\title{
Resíduos de Serviços de Saúde (RSS): Uma abordagem qualitativa
}

\author{
Claudio Fernando Mahler' ${ }^{1}$ Leonardo de Lima Moura² \\ cfmahler@gmail.com, leonardodelmoura@gmail.com \\ ${ }^{1}$ GETRES/PEC/COPPE, Universidade Federal do Rio de Janeiro, 68506, Rio de Janeiro, Brasil \\ ${ }^{2}$ GETRES/PEC/COPPE, Universidade Federal do Rio de Janeiro, 68506, Rio de Janeiro, Brasil
}

DOI: 10.17013/risti.23.46-6o

\begin{abstract}
Resumo: O gerenciamento inadequado de Resíduos de Serviços de Saúde (RSS) leva a inúmeros problemas de saúde à população, os quais ocorrem em todo o mundo, em especial o menos desenvolvido. Apoiado em técnicas de pesquisa qualitativa, avaliou-se o funcionamento de um hospital quanto aos RSS por meio de entrevistas com stakeholders, funcionários com postos chaves na estrutura de comando e o pessoal de operação, além da observação diária das atividades. Diversas discrepâncias foram observadas entre os discursos e a prática. Foi observado o desconhecimento destes profissionais em relação a diversos aspectos relacionados ao gerenciamento de RSS o que impacta significativamente a implementação de um gerenciamento correto. Os resultados permitiram concluir que o gerenciamento de RSS tem algumas deficiências o que eleva consideravelmente o potencial risco dos resíduos dos serviços de saúde ao meio ambiente e à saúde pública.
\end{abstract}

Palavras-chave: resíduos de serviços de saúde; pesquisa qualitativa; hospital maternidade; entrevistas; avaliação

\section{Healthcare waste (HCW): A qualitative approach}

Abstract: Inadequate healthcare waste management (HCWM) leads to numerous health problems for the population, which occur worldwide, especially the least developed. Supported by qualitative research techniques, a hospital's performance on HCWM was assessed through interviews with key personnel in the command structure and operational personnel, as well as daily observation of activities. Several discrepancies were observed between discourses and practice. It was observed the lack of knowledge of these professionals in relation to several aspects related to the HCWM, which significantly impacts the implementation of a correct management. The results allowed to conclude that the HCWM has some deficiencies which considerably increases the potential risk of health services wastes to the environment and to public health

Keywords: healthcare waste management; qualitative research; maternity; interviews; assessment. 


\section{Introdução}

O controle de qualidade e desempenho constitui uma das maiores preocupações em unidades de saúde. Fernandes e Tareco (2016), numa revisão sobre o tema, observaram a relevância dos Sistemas de InformaçãoI como um suporte às práticas de saúde, funcionando como uma ferramenta de apoio à gestão. No entanto, há ainda ausência de sistemas de informação no que se refere ao gerenciamento de Resíduos de Serviços de Saúde (RSS).

O gerenciamento de RSS se configura como um problema atual em muitos países em desenvolvimento já que ao mesmo tempo em que lidam com o aumento populacional e da demanda por serviços de saúde devido a maior expectativa de vida da população, com consequente aumento de doenças crônicas. Além disso, enfrentam com freqüência períodos de dificuldades financeiras, associados nestes países a falhas em diversas etapas do manejo dos RSS, principalmente no que se refere à segregação e disposição final adequada destes resíduos.

Apesar das práticas de gerenciamento de RSS variarem de país para país já que dependem de diversos fatores como: as condições sócio econômicas, os recursos humanos e financeiros disponíveis e as legislações existentes, uma das etapas primordiais para implementação de um processo adequado de gerenciamento de RSS é o conhecimento, por parte dos gestores, da quantidade de RSS gerada e sua composição.

Além disso, é indispensável que seja preconizado na instituição a adequada segregação dos RSS pelos profissionais de saúde já que caso os resíduos infectantes, devido as suas características patogênicas, não forem manipulados de forma adequada, estes podem se configurar como potencial risco ao meio ambiente e à saúde pública.

A preocupação com as questões ambientais começou a despertar preocupação em diversos stakeholders, desde organizações não governamentais ( $\left.\mathrm{ONG}^{\prime} \mathrm{s}\right)$, governos até a sociedade em geral. Consequentemente, tem mobilizado os diversos atores com a finalidade de implementar ações para mitigar esses impactos negativos ao meio ambiente. (Severo \& Sousa, 2016).

Apesar disso, no Brasil o gerenciamento de RSS ainda se configura como um problema como demonstrado por Von Sperling \& de Vasconcelos Barros (2014), num estudo envolvendo 53 estabelecimentos de saúde, no qual observaram que procedimentos simples como a identificação das entradas dos abrigos e dos sacos plásticos não era realizada na maior parte dos estabelecimentos. Além disso, num número considerável de instituições de saúde, os RSS eram armazenados diretamente sobre o piso.

Falhas como a descrita por Von Sperling \& de Vasconcelos Barros (2014) foram também observadas por Maders \& Cunha (2015) num hospital de emergência de Macapá/ Amapá que também destacaram a falta de infraestrutura adequada e de rotinas para o manejo dos RSS. Da Silva André \& Takayanagui (2016) ressaltaram principalmente, num estudo realizado em estabelecimentos de saúde de Ribeirão Preto/ São Paulo, o desconhecimento por parte dos responsáveis pelo gerenciamento de RSS quanto aos resíduos gerados.

É importante destacar que o desconhecimento por parte dos profissionais responsáveis pelo gerenciamento de RSS impacta diretamente no processo de capacitação dos demais 
profissionais de saúde. Amarante et al. (2016) destacam as falhas na formação acadêmica destes profissionais e a inexistência de cursos de aperfeiçoamento como as principais responsáveis para que haja contínuas falhas em todas as etapas do gerenciamento de RSS. Mahler \& Moura (2017) também observaram algumas discrepâncias entre os discursos de profissionais envolvidos no gerenciamento de RSS de um hospital do Estado do Rio de Janeiro e as atividades que eram executadas.

Ciente da importância de avaliar o conhecimento dos profissionais responsáveis pelo gerenciamento de RSS a cerca das práticas adotadas na instituição, o presente artigo buscou traçar um diagnóstico do gerenciamento de RSS por meio de uma pesquisa qualitativa envolvendo a utilização do instrumento intitulado Gerenciamento de Resíduos de Serviços de Saúde- Instrumento de avaliação rápida proposto pela OMS e cuja versão brasileira foi traduzida e validada por Silva (2011). Ao mesmo tempo, buscou-se por meio da pesquisa de campo, outra ferramenta usual da pesquisa qualitativa, analisar se as informações fornecidas pelo diretor do hospital, pela profissional responsável pelo Plano de Gerenciamento de Resíduos de Serviços de Saúde (PGRSS) e enfermeira chefe da Comissão de Controle de Infecção Hospitalar (CCIH) responsável pela capacitação dos demais profissionais eram fidedignas ao que ocorria na prática.

\section{Metodologia}

A presente pesquisa pode ser classificada como qualitativa, pois, segundo Minayo (2011), tais pesquisas focam em questões muito específicas, voltadas para significados, aspirações, atitudes e pensamentos. Fatos estes que não podem ser facilmente operacionalizados por meio de variáveis. O caso da presente pesquisa compreendeu a avaliação do gerenciamento de RSS por meio de entrevistas e observação direta da rotina hospitalar. Além disso, buscou atender algumas características destacadas por Stake (2016) como fundamentais para o estudo qualitativo:

1. Carater interpretativo: Os pesquisadores na execução do projeto de pesquisa buscaram interações com os profissionais envolvidos no gerenciamento de RSS, vivenciando suas realidades e baseando suas descobertas na interação com os mesmos;

2. Carater experiencial: Por meio da inserção na rotina de trabalho dos profissionais de limpeza, os autores buscaram enfocar as percepções dos próprios profissionais envolvidos no manejo de RSS em relação às suas práticas por meio da observação direta, escuta e realização de entrevistas;

3. Carater situacional: $\mathrm{O}$ foco da pesquisa foi compreender as características referentes ao sistema de gerenciamento de RSS de uma maternidade e correlacionar com a percepção tanto dos profissionais de saúde quanto dos profissionais de limpeza daquela instituição.

4. Carater personalístico: Buscou analisar as percepções individuais de cada um dos profissionais analisados e por fim traçar um diagnóstico do gerenciamento de RSS frente a estas perecepções.

Seguindo a divisão da pesquisa qualitativa descrita por Minayo (2011), o projeto de pesquisa foi dividido em três etapas: fase exploratória, pesquisa de campo e análise e tratamento do material documental. 
Outro fator que destaca o carater qualitativo da pesquisa apresentada é o fato desta ser descritiva, pois teve o intuito assim como salientado por Gibbs (2009) de responder o questionamento "O que está ocorrendo aqui”, no caso da pesquisa analisar amplamente as características de um determinado grupo de análise, no caso o setor de gerenciamento de RSS de estabelecimentos de saúde. Para a escolha do método de pesquisa aqui empregado, utilizou-se como base o questionamento a que o artigo se propõe a responder que foi:

"Como é o processo de gerenciamento de RSS da instituição de saúde analisada frente à legislação vigente?" Yin (2010) destaca que questões envolvendo "como" e "por que" apresentam característica de pesquisa mais explanatória, favorecendo a utilização de métodos como experimentos, pesquisas históricas e estudos de caso.

Como as pesquisas direcionadas à análise do gerenciamento de RSS têm como finalidade que as situações observadas no processo de gerenciamento sejam as mais fidedignas possíveis das rotinas e o foco em situações contemporâneas é algo inerente do próprio objetivo destas pesquisas, a adoção da metodologia estudo de caso foi a mais indicada.

Para traçar este diagnóstico do conhecimento dos gestores a cerca do gerenciamento de RSS, utilizou-se o instrumento intitulado Gerenciamento de Resíduos de Serviços de Saúde- Instrumento de avaliação rápida proposto pela Organização Mundial de Saúde (OMS) e cuja versão brasileira foi traduzida e validada por Silva (2011). Em virtude da abrangência deste instrumento, optou-se pelo uso apenas da ferramenta D que possui um escopo local e apresenta questionários voltados para obtenção de dados dos funcionários da instituição de saúde analisada, a saber: D-2 e D-3, ou seja, gestor do estabelecimento de saúde, enfermeira chefe/Comissão de Controle de Infecção Hospitalar (CCIH) e responsável pelo Gerenciamento de RSS.

Para a realização de entrevistas, submeteu-se o projeto de pesquisa ao Comitê de Ética e Pesquisa (CEP) da Universidade Federal do Rio de Janeiro e após a aprovação sob CAAE: 45388415.7.0000.5257 as entrevistas foram agendadas de acordo com a disponibilidade dos profissionais com o intuito de minimizar seu impacto nas suas atividades diárias.

Antes do início das entrevistas, foi disponibilizado o Termo de Consentimento Livre e Esclarecido (TCLE) e o instrumento de coleta de dados para que o entrevistado pudesse realizar uma leitura prévia e sanar eventuais dúvidas. Os tópicos questionados foram colocados de forma aberta aos entrevistados sem que houvesse nenhum direcionamento ou pressão para uma determinada resposta. Contudo, questionamentos considerados relevantes pelo pesquisador no andamento da entrevista e que não estivessem contemplados no questionário foram abordados. As entrevistas ocorreram na própria sala de trabalho dos funcionários em horário previamente estabelecido.

No intuito de avaliar a fidedignidade das informações obtidas, foi realizada a pesquisa de campo por meio da observação diária do processo de trabalho dos profissionais de limpeza envolvidos no manejo de RSS durante o período de janeiro e fevereiro, excetuando-se fins de semana, com um foco direcionado para os coletores que são os profissionais responsáveis pela coleta, transporte e armazenamento de RSS.

Com o intuito de minimizar a visão do pesquisador como "agente fiscalizador" foi realizado um amplo esclarecimento do objetivo do trabalho com a finalidade de que os 
profissionais envolvidos no gerenciamento pudessem ter uma melhor compreensão da pesquisa e sanassem eventuais dúvidas.

Quanto à obtenção de informações, privilegiou-se a observação, a escuta e anotação dos relatos espontâneos dos profissionais de limpeza deixando a cargo destes a descrição das suas atividades e rotinas, buscando-se dessa forma tentar minimizar a influência do pesquisador nas suas atividades.

Para o registro das informações, utilizou-se um diário de pesquisa onde foram realizadas anotações indicando o dia em que foram coletadas e posteriormente transcritas para o Word. No caso de relato espontâneo estabeleceu-se um código de identificação do autor do relato de forma a garantir o seu anonimato, como a utilização das três primeiras letras da função e um número, como por exemplo: COL 1. Também se utilizou a foto documentação dos fatos de forma a comprovar as informações obtidas

\section{Resultados e Discussão}

Os principais resultados e comentários em relação às entrevistas foram subdivididos em seções de acordo com o instrumento de coleta de dados na seção abaixo.

\subsection{Entrevista com o gestor do estabelecimento de saúde}

Na tabela abaixo, são apresentados alguns itens do questionário nos quais a resposta do entrevistado se mostrou uma fonte de evidência para corroborar as ferramentas de coleta de dados previamente utilizadas.

\section{Item do questionário}

Tipo de estabelecimento e nível de hierarquia

Número de leitos

Treinamento/Capacitação

Treinamento da responsável pelo PGRSS

Comissão de RSS

Alocação orçamentária para GRSS

\section{Trecho da fala do entrevistado}

"Na prática o hospital pode ser considerado de alta complexidade, pois atende a todas as pacientes que vem para cá”

Existe uma sobreocupação que gira em torno de $120 \%$.

"Treinamento de PGRSS, mas você pode obter maiores informações diretamente com a Comissão de Controle de Infecção Hospitalar (CCIH)"

"Único treinamento que recebeu foi por parte da OSs para padronizar o PGRSS..."

"Toda a parte de resíduos fica a cargo da CCIH......"

“.....deve haver um direcionamento para diminuição deste percentual em virtude da escassez de recursos financeiros."

Tabela 1 -Principais relatos do gestor do hospital 
Segundo o gestor da instituição o nível de hierarquia a que pertence o hospital nos dados do Cadastro Nacional de Estabelecimento de Saúde (CNES) é de instituição de média complexidade, porém na prática esta pode ser classificada como de alta complexidade com base no seguinte argumento do entrevistado:

"Na prática o hospital pode ser considerado de alta complexidade, pois atende a todas as pacientes que vem para cá, deveria ser um hospital porta fechada o que diminuiria a complexidade dele, no entanto não é isto que ocorre na prática. Noutro dia, houve até um paciente baleado aqui e ele não está de todo modo errado, viu que era um hospital e entrou. Existe um controle maior no ambulatório, mas no hospital não.”

$\mathrm{Na}$ fala apresentada pelo gestor pode se destacar o fato de que contrariamente ao que estabelecido na teoria de só atender pacientes referenciadas ou que realizem acompanhamento no hospital, no dia-a-dia o hospital acaba por atender todos os tipos de paciente que se dirijam à instituição, exemplificado pela ida de um paciente baleado para o local.

O fato de o hospital ser basicamente "porta aberta" principalmente para gestantes pode ser uma das explicações para a resposta do gestor quando questionado em relação ao número de leitos:

"Existe uma sobreocupação que gira em torno de 120\%. Em termos de leitos fixos são 78 leitos, considerando verde, lilás e UI No entanto, no total são 98."

Nesta declaração, o entrevistado estabelece em números o que foi observado durante o período de investigação, quantificação e a caracterização gravimétrica dos setores, principalmente do acolhimento no qual se encontrou absorventes pós-parto que é o fato do hospital estar atendendo acima da sua capacidade de atendimento.

Além de influenciar nas características qualitativas dos RSS de cada setor já que o setor de acolhimento passou a ser voltado apenas para atendimento emergencial passou a realizar partos, a ocupação acima da capacidade influencia consideravelmente na quantidade de RSS gerada impedindo que se consiga estabelecer acuradamente uma média de geração de RSS no hospital dificultando o dimensionamento do sistema de gerenciamento de RSS.

No item Equipe/ profissionais, de acordo com gestor administrativo, o treinamento/ capacitação contempla o gerenciamento de RSS e isto fica a cargo da CCIH cuja enfermeira chefe também é responsável pelo gerenciamento de RSS. Quantos aos tipos de treinamento/capacitação oferecidos, ele respondeu da seguinte forma:

"Treinamento de PGRSS, mas você pode obter maiores informações diretamente com a CCIH."

Na declaração do gestor administrativo este ressalta apenas o treinamento de PGRSS, documento que como já analisado anteriormente apresenta diversas inadequações à legislação vigente. $\mathrm{O}$ interessante é que o administrativo não se alonga em relação aos treinamentos deixando demais informações a cargo da própria CCIH o que pode indicar uma falta de conhecimento no que se refere às capacitações/treinamentos ofertados pela instituição.

Quando questionado se a responsável pelo GRSS recebeu algum tipo de treinamento, ele afirmou: 
"Único treinamento que recebeu foi para padronizar o PGRSS, se ela recebeu algum tipo de capacitação foi por conta própria."

Segundo gestor administrativo, houve apenas um treinamento para que a profissional estabelecesse um PGRSS padrão a outras instituições gerenciadas pela OSs. O mero treinamento focado na padronização do PGRSS é inadequado quando se espera que este documento seja elaborado de acordo com as características da instituição em que vai ser aplicado. Além disso, deveria ser um objetivo da unidade primar pela capacitação contínua dos seus funcionários, principalmente daquele que é responsável pela capacitação dos demais colegas.

As respostas referentes a transporte de RSS para local externo foram tipo padrão e estavam de acordo com a realidade observada. É importante destacar que o gestor administrativo é o responsável por gerenciar os contratos na instituição com as empresas de coleta externa.

No item regulamentação para GRSS, quando questionado se havia problemas quanto à aplicação de regulamentações nacionais/ estaduais de GRSS, a resposta negativa do gestor administrativo com a anuência do diretor contradiz as dificuldades normalmente associadas à adoção da RDC 306/04 relatadas por profissionais de saúde e gestores noutras pesquisas.

A negativa a esta resposta motivou uma pergunta relacionado à como funcionava a comissão de resíduos presente no PGRSS na qual consta o gestor da instituição como membro, o que levou o mesmo a dar seguinte resposta:

"Toda a parte de resíduos fica a cargo da CCIH e fica a meu cargo cobrar para que seja executado o que foi proposto."

A declaração do gestor do estabelecimento indica mais claramente o que já vinha sendo demonstrado pelo gestor administrativo que tudo o que se refere a RSS está sob responsabilidade da CCIH o que indica que não há uma participação efetiva dos demais setores no gerenciamento de RSS o que faz com que, mesmo como integrante da comissão, a sua função seja apenas cobrar a execução do que foi estabelecido.

No item voltado para política /orçamento embora o gestor administrativo e o diretor da instituição afirmarem que a alocação orçamentária para a Gestão de Residuos de serviços de Saúde (GRSS) é suficiente, em virtude das características deste tipo de administração estes não souberam informar o percentual destinado ao gerenciamento de RSS, segundo o seguinte motivo apresentado pelo gestor administrativo:

"Isto fica a cargo da administração da OSs, mas deve haver um direcionamento para diminuição deste percentual em virtude da escassez de recursos financeiros"

Com base na fala do gestor administrativo, nota-se que neste modelo de administração a direção da OSs é a responsável por alocar os recursos financeiros direcionados para o gerenciamento de RSS em cada uma das instituições sob seu comando. É importante ressaltar na declaração do gestor que a falta de recursos financeiros poderia motivar uma redução deste percentual.

Comparando-se a fala deste profissional com a realidade apresentada no hospital, pode-se afirmar que a alocação orçamentária era extremamente insatisfatória uma fez 
que como já previamente relatado havia contêineres danificados, tamanho reduzido do abrigo externo e portas e telas de proteção deste local danificadas. Uma maior redução no orçamento deste setor impactaria ainda mais negativamente no gerenciamento de RSS.

\subsection{Entrevista com a enfermeira chefe/CCIH}

Na tabela 2 constam os itens e trechos das falas que forneceram evidências sobre o gerenciamento de RSS, facilitando a triangulação dos resultados obtidos nas outras etapas

\begin{tabular}{ll}
\hline Item & Trecho da fala da entrevistada \\
\hline Número de leitos & $\begin{array}{l}\text { "Não é fácil precisar, mas pode colocar que está } \\
\text { superlotado." }\end{array}$ \\
\hline Taxa média de ocupação e pacientes atendidos & "Infelizmente não há informações atualizadas.....” \\
\hline Acidentes perfurocortantes & "Geralmente são 2 a 3 casos por ano." \\
\hline Sistema de cores de RSS & $\begin{array}{l}\text { "Meio complicado de lembrar estas cores porque } \\
\text { cada hospital trabalha de uma forma” }\end{array}$ \\
\hline Transporte interno e coleta & $\begin{array}{l}\text { "Quando há a passagem de comida, não passa } \\
\text { resíduo.” }\end{array}$ \\
\hline Regulamentações para GRSS & "....logo que acaba a reunião volta tudo a como \\
\hline
\end{tabular}

Tabela 2 - Principais relatos da enfermeira chefe

No que se refere ao número de leitos ocupados, a resposta da profissional de saúde é parecida com a do diretor do hospital, embora apresente maior imprecisão:

"Esta é uma pergunta difícil de responder, o hospital normalmente está superlotado. Exemplo na UI, teve vez que eu tendo 8 leitos tinha 12 crianças. Não é fácil precisar, mas pode colocar que está superlotado."

Quanto à lotação do hospital, nota-se que a profissional de saúde apesar de não conseguir precisar a quantidade de sobreocupação, busca exemplificar o que tem sido observado na instituição como a manutenção de 4 crianças a mais na UI.

Quando questionada em relação à média de ocupação e o número de pacientes ambulatoriais atendidos por dia, novamente ela demonstrou imprecisão afirmando que eram respectivamente $100 \%$ e 800 . Em relação aos dados referentes aos atendimentos ambulatoriais ela afirmou que estes dados eram referentes ao ano anterior e deu a seguinte justificativa para ausência de dados recentes:

"Infelizmente não há informações atualizadas, pois a pessoa responsável saiu de férias e ainda não me passou os dados, posso te afirmar do ano passado."

A imprecisão de informações referentes ao número de leitos ocupados na instituição e o número de pacientes atendidos influencia diretamente no gerenciamento de RSS, uma vez que como há uma correlação positiva entre o número de pacientes e a geração 
de RSS, o desconhecimento desses dados dificulta a implementação de um adequado PGRSS na instituição.

No item equipe/profissionais de saúde, a enfermeira destacou que todos os profissionais de saúde são imunizados em relação à hepatite e tétano. No entanto, não soube precisar quantos acidentes com perfurocortantes ocorreram nos últimos meses, mas deu a seguinte resposta:

“Geralmente são 2 a 3 casos por ano.”

Sobre as medidas adotadas, ela descreveu o seguinte procedimento:

"Dependendo do acidente, vê se precisa tomar medicação ou não. O Serviço Especializado em Engenharia de Segurança e em Medicina do Trabalho (SESMT) conversa com as pessoas, na integração o funcionário é treinado.”

Embora não sejam dados atualizados, nota-se que há, segundo informações da enfermeira, poucos casos de acidentes perfurocortantes no hospital o que pode ser reflexo do processo de capacitação citado por ela ou de subnotificação dos casos já que foram encontradas seringas com agulhas desencapadas entre os RSS do grupo D. Quanto aos procedimentos adotados, observa-se o destaque dado ao SESMT para evitar a ocorrência de novos acidentes.

No item recipiente de acondicionamento/ armazenamento, apesar da enfermeira ter afirmado que existe um sistema diferenciado de cores por tipo de RSS, a mesma apresentou dificuldade para descreve-lo como pode ser visto na sua resposta:

"Branco para infectante e cinza para comum. Meio complicado de lembrar estas cores porque cada hospital trabalha de uma forma, lá no outro hospital acho que o comum éverde."

Apesar de ser a própria profissional responsável por ter estabelecido o PGRSS e as cores de identificação dos RSS, esta apresenta dificuldade de lembrar que a cor dos sacos estabelecida na instituição para resíduo comum é preta. O interessante é que ela atribui esta dificuldade a diversos tipos de padronizações estabelecidos pelos hospitais, inclusive no uso da cor verde noutro hospital pertencente a OSs.

No item referente ao transporte interno, a profissional de saúde quando questionada afirmou que as práticas atuais de transporte interno e coleta de RSS são dotadas de segurança suficiente e justificou o motivo da sua resposta:

“Quando há a passagem de comida, não passa resíduo.”

A fala da enfermeira, conforme já descrito anteriormente, não condiz totalmente com a realidade existente no hospital uma vez que como os horários de coleta não são padronizados na instituição pode haver a possibilidade de fluxo de alimentos e resíduos ao mesmo tempo.

Quanto ao item regulamentações para GRSS, a exposição do PGRSS, embora seja feita na instituição sofreu alterações quanto a periodicidade por influência do pesquisador, pois antes este era feito de forma anual e após a conversa com pesquisador houve sugestão que passasse a ser mais vezes ao ano então foi alterado para semestral. 
No entanto, a enfermeira relatou haver ainda muitos problemas no que se refere à efetividade desta exposição, conforme relato abaixo:

"Tenho muitas dificuldades em relação ao treinamento, pois falo com as pessoas sobre a questão de RSS, mas logo que acaba a reunião volta tudo a como era antes.”

Sobre o relato da enfermeira, pode-se destacar a percepção por parte dela da ineficiência dos treinamentos executados na instituição, o que parece indicar a necessidade não apenas de alterar a frequência dos treinamentos, mas também a forma como este feito e o método de exposição das informações.

Além disso, parece ser indispensável que haja uma sensibilização constante dos profissionais de saúde principalmente no que se refere a adequada segregação de RSS, por exemplo, por meio de pequenas reuniões de curta duração no próprio setor mais direcionadas para apresentação dos principais conceitos da legislação e respostas de eventuais dúvidas dos profissionais.

Apesar do relato da enfermeira sobre a realização de treinamentos voltados para o PGRSS do hospital, não é a mesma que administra os treinamentos dos funcionários da empresa terceirizada já que estes são capacitados pela enfermeira da própria empresa. Sobre estes treinamentos, COL 1 deu os seguintes relatos em diversas ocasiões:

"O hospital dificilmente faz treinamento dos funcionários, acaba deixando a cargo da empresa."

"Não tem treinamento específico para resíduo, a empresa segue o que hospital faz."

"Não recebi treinamento aqui, a minha forma de trabalhar trouxe de outros lugares"

Sobre as afirmativas acima de diferentes formas o coletor relata a carência de treinamentos voltados para resíduos na instituição além da terceirização dos treinamentos a cargo da enfermeira da empresa terceirizada. Embora saliente o fato destes treinamentos estarem em consonância com a rotina do estabelecimento de saúde, destaca a falta de enfoque a resíduos nesta capacitação, ressaltando que o modo que executa suas atividades é de acordo com a experiência prévia na função.

O outro coletor quando questionado sobre a sua forma de trabalho, respondeu da seguinte forma:

"Já entrei como coletor me explicaram qual era o branco e o preto, onde ficava os contêineres, necessidade de lavagem do abrigo das paredes. O serviço que me ensinaram foi de auxiliar de serviços gerais."

Em relação ao relato acima, pode-se ressaltar que este coletor relata que houve certa enumeração das atividades que deveria executar e também uma explicação básica da localização dos contêineres e do que estes deveriam armazenar. No entanto, o mesmo conclui que não houve qualquer tipo de orientação específica para sua atividade de coletor, sendo ministradas informações semelhantes a que eram passadas à auxiliar de serviços gerais.

Isto comprova a falta de padronização na atividade dos coletores observada no hospital, fazendo que cada coletor realize a sua função da mesma forma que executava em outras instituições de saúde, tornando cada plantão função do coletor. Um fato que exemplifica 
isto, é que num plantão havia varrição de toda área externa do hospital enquanto no outro não.

Embora destacassem que esta era uma das atividades do coletor, quando questionado em relação ao motivo que executava esta tarefa, o coletor respondeu que no antigo hospital que trabalhava, ele era responsável pela varrição externa.

\subsection{Enfermeira responsável pelo PGRSS}

Após o término da entrevista com a enfermeira chefe da CCIH, como esta era também a responsável pelo PGRSS, a segunda entrevista foi realizada e os principais itens e trechos da fala são destacados na tabela 3 .

\begin{tabular}{ll}
\hline Item & Trecho da fala da entrevistada \\
\hline Geração de RSS do grupo A e D & $\begin{array}{l}\text { "Estranho, eu tenho muito mais biológico do } \\
\text { que comum.” }\end{array}$ \\
\hline Geração de resíduos químico farmacêutico & “.......... quase não gera” \\
\hline Geração de resíduos químicos & “Mesma coisa, quase não gera” \\
\hline Área de armazenamento de RSS & “A arquitetura dela não é totalmente segura por \\
não haver algumas coisas como exaustor.......”
\end{tabular}

Tabela 3 - Principais relatos da enfermeira responsável pelo PGRSS

No que se refere a geração de RSS, a enfermeira salientou que todas estas informações estavam presentes no PGRSS, com exceção de resíduos químicos-farmacêuticos, resíduos químicos e rejeitos radioativos que não eram gerados na instituição.

No PGRSS há apenas dados referentes aos RSS do grupo A e D. Segundo o PGRSS, o hospital gera diariamente 131.040 litros (L) de RSS do grupo A enquanto os RSS do grupo D são subdivididos em resíduos de cozinha e resíduos administrativos, gerando diariamente respectivamente 960 L e 8.223 L. Após enunciar os valores, a enfermeira emitiu a seguinte declaração:

“Estranho, eu tenho muito mais biológico do que comum.”

Analisando-se a declaração, nota-se o desconhecimento por parte da enfermeira em relação aos dados de geração existentes no PGRSS. Isto demonstra que estes dados provavelmente não foram determinados de forma precisa e avaliados quanto a sua autenticidade antes de serem redigidos no PGRSS.

Comprova-se isto analisando a métrica estabelecida normalmente por empresas terceirizadas e hospitais que é a quantificação de RSS por contêiner, tendo como base um contêiner de $240 \mathrm{~L}$.

Dividindo-se a geração diária por 240 L se obteria no que se refere a RSS do grupo A valores que demonstram que estes dados não foram obtidos por qualquer tipo de medição já que segundo contrato com a empresa de coleta externa, a coleta diária normalmente era de 9 contêineres. 
Quanto aos resíduos comuns, haveria a necessidade diária de coleta de 35 contêineres, valor acima do estabelecido com a empresa terceirizada de 26 contêineres e dos valores de 17 contêineres normalmente coletados. Há, portanto, uma certa desorganização neste quesito.

Já em relação a geração de resíduos químicos- farmacêuticos, houve a seguinte resposta:

"Os medicamentos próximos de vencer são encaminhados para outros hospitais e em virtude disso, quase não gera."

A declaração da enfermeira de que quase não gerava esse tipo de resíduo não é um fato impeditivo para se estabelecer o gerenciamento adequado desse grupo de RSS já que este acaba sendo alvo de segregação inadequada pelos profissionais de saúde. Isto foi comprovado pela observação da presença de frascos de medicamentos, RSS do grupo B, misturado nos RSS do grupo D. O fato da profissional responsável pelo PGRSS desconhecer a existência da geração de RSS do grupo B no hospital é preocupante, uma vez que ela é a responsável por realizar avaliações periódicas do PGRSS implantado no que se refere a indicadores e por capacitar os profissionais de saúde no que se refere ao manejo adequado de RSS.

Ao ressaltar o encaminhamento de medicamentos próximos ao vencimento para outros estabelecimentos de saúde, a enfermeira abordou apenas um exemplo de RSS do grupo B possível de serem gerados que são os resíduos de medicamentos vencidos. Com isso, a profissional desconsiderou que frascos de medicamentos vazios, típicos do grupo B, são gerados diariamente na unidade hospitalar sem haver qualquer informação aonde estes são segregados.

Quando questionada em relação aos resíduos químicos exemplificados por saneantes, desinfetantes e antissépticos, a responsável pelo PGRSS respondeu de forma parecida:

"Mesma coisa, quase não tem, acaba aproveitando em outras unidades.”

Quanto aos resíduos químicos, há uma repetição da resposta da profissional responsável pelo PGRSS. Em virtude disso, não há uma estimativa da sua geração e nem o estabelecimento de um manejo adequado para estes tipos de resíduos. A consequência disso é a segregação inadequada destes tipos de RSS, já que se notou a presença de antisséptico, RSS do grupo B, misturado com RSS do grupo D.

Quanto ao motivo da pequena geração desse tipo de resíduo, novamente, a responsável pelo PGRSS apresenta uma visão focada em RSS do grupo B próximos a vencimento sem se atentar que o antisséptico é utilizado diariamente na realização de curativos em parturientes.

A falta de informações referentes à geração de RSS do grupo B no estabelecimento hospitalar impede que haja a adoção de medidas proativas que busquem minimizar os impactos negativos oriundos do manuseio inadequado destes tipos de RSS, especialmente se considerarem os impactos negativos ao meio ambiente associados aos RSS do grupo B o que faz com que a RDC 306/04 estipule a necessidade de tratamento prévio, antes da disposição final destes resíduos.

Em relação ao item segregação, no questionamento de que EPIs dispõe a equipe que manuseia os RSS, a enfermeira respondeu que os profissionais de limpeza utilizavam luvas, botas, avental, calças compridas, máscaras e óculos de proteção. 
No entanto, a utilização de todos estes EPIs só foi observada nos coletores, enquanto que as demais profissionais de limpeza só utilizavam luvas, botas e calças compridas. Isto foi corroborado pela resposta do COL 1 quando questionado se observava risco na sua atividade:

"As meninas que estão mais expostas, pois elas que manipulam o lixo. Tem risco de respingar sangue e não tem óculos, muito mais um avental."

Nota-se na resposta do coletor que este caracteriza as atividades das profissionais de limpeza como de maior risco, já que tem que manipular os sacos de RSS abertos e realizar o seu fechamento.

Apesar disso, há ausência entre as profissionais de limpeza de equipamentos de proteção básicos para minimizar os riscos ocupacionais.

No item referente à área de armazenamento de RSS, quando questionada se a área de armazenamento de RSS poderia ser considerada segura, a enfermeira respondeu de forma afirmativa. No entanto, acrescentou a seguinte declaração:

“A arquitetura dela não é totalmente segura por não haver algumas coisas como exaustor, mas pelo menos não expõe o coletor ao risco."

Com base na resposta da responsável pelo PGRSS, pode-se destacar que nem mesmo por ela esta área pode ser classificada como segura, salientando que apesar de não expor o coletor ao risco há ainda alguns fatores que estão em desacordo com o estabelecido pela legislação como, por exemplo, a inexistência de exaustor no abrigo. Acrescente-se a isso o fato de que não há também uma área específica para higienização dos carrinhos de coleta e o fato do abrigo externo estar localizado ao lado do gerador do hospital e quando o gerador se encontrava em funcionamento, permanecer no setor era muito difícil devido ao barulho excessivo.

Comojá citado e discutido anteriormente a entrevistada mostrou amplo desconhecimento em relação ao tratamento de RSS e disposição final dos RSS já que não soube responder nenhum dos questionamentos realizados

\section{Conclusões}

Com base nas entrevistas realizadas com o diretor da instituição e a enfermeira chefe e responsável pelo gerenciamento de RSS, nos relatos dos profissionais de limpeza envolvidos no manejo de RSS e na observação diária apoiado na pesquisa qualitativa pode-se constatar que existem falhas nas diversas etapas do gerenciamento de RSS. Comparando os discursos da profissional de saúde com os relatos dos funcionários de limpeza e com a realidade observada, várias discrepâncias foram notadas entre a fala dos entrevistados e a fala dos funcionários de limpeza associada ao que efetivamente ocorria diariamente no hospital. Tal resultado é um indicativo de que há falhas no processo de implementação e operação do PGRSS e capacitação contínua dos funcionários envolvidos no manejo de RSS o que consequentemente aumenta o potencial de risco destes resíduos à saúde pública, à saúde ocupacional e ao meio ambiente. 
Quanto à adequação do gerenciamento de RSS à legislação vigente, questionamento principal deste artigo, o instrumento de coleta de dados utilizado, com base na pesquisa qualitativa, permitiu determinar que diversos parâmetros preconizados pela legislação não são adotados na instituição, muitas vezes devido ao desconhecimento da profissional responsável. O presente trabalho também se configura como uma importante contribuição na utilização de pesquisa qualitativa para avaliar o processo de gerenciamento de RSS em estabelecimentos de saúde e comparativamente a outros trabalhos, focados na pesquisa quantitativa, desenvolvidos sobre o tema que obtiveram resultados semelhantes no que se refere à inadequação do gerenciamento de RSS em unidades de saúde no Brasil.

\section{Referências}

Amarante, J. A. S., Rech, T. D., \& Siegloch, A. E. (2016). Management assessment of drug waste and other health care waste in Upland Region of Santa Catarina, Brazil. Engenharia Sanitaria e Ambiental, 22(2).

Da Silva André, S. C., \& Takayanagui, A. M. M. (2016). Geração de Resíduos de Serviços de Saúde em Hospitais do Município de Ribeirão Preto-SP. Engenharia Sanitária e Ambiental, 21(1).

De Souza Minayo, M. C. (2011). Pesquisa social: teoria, método e criatividade. Petrópolis: Editora Vozes Limitada.

Fernandes, S., \& Tareco, E. (2016). Sistemas de informação como indicadores de qualidade na saúde: Uma revisão de níveis de abordagem. RISTI-Revista Ibérica de Sistemas e Tecnologias de Informação, (19), 34-45.

Gibbs, G. (2009). Análise de dados qualitativos: coleção pesquisa qualitativa. Porto Alegre: Bookman Editora.

Maders, G. R., \& Cunha, H. F. A. (2015). Análise da gestão e gerenciamento dos resíduos de serviços de saúde (RSS) do Hospital de Emergência de Macapá, Amapá, Brasil. Engenharia Sanitária e Ambiental, 20(3), 379-388.

Mahler, C., \& Moura, L. (2017). Avaliação do gerenciamento de Resíduos de Serviços de Saúde (RSS) de um hospital maternidade do Estado do Rio de Janeiro. In Atas- Investigação Qualitativa em Engenharia e Tecnologia do CIAIQ 2107Vol. 4 - ISBN: 978-972-8914-78-3.

Severo, E. M., \& Sousa, H. J. (2016). Avaliando a Sustentabilidade das Edificações através de Ferramentas Qualitativas e Quantitativas. RISTI-Revista Ibérica de Sistemas e Tecnologias de Informação, (19), 01-14.

Silva, E. N. C. (2011). Gerenciamento de Resíduos de Serviços de Saúde: adaptação transcultural e validação do instrumento Health-care Waste Manegement - Rapid Assessment Tool.2011. Tese (Doutorado) - Escola Nacional de Saúde Pública, Rio de Janeiro.

Stake, R. E. (2016). Pesquisa qualitativa: estudando como as coisas funcionam. São Paulo: Penso Editora. 
Von Sperling, E., \& de Vasconcelos Barros, R. T. (2014). Avaliação do gerenciamento dos resíduos de serviços de saúde em municípios da região metropolitana de Belo Horizonte (Brasil). Engenharia Sanitária e Ambiental, 19(3).

Yin, R. (2010). Estudo de caso: planejamento e métodos. Porto Alegre: Bookman Editora. 